九州大学学術情報リポジトリ

Kyushu University Institutional Repository

\title{
Studies on Potato virus $Y$ Isolates Infecting Potato and Tobacco in Korea
}

Choi, Hong-Soo

Bhat, A-Ishwara

Division of Crop Protection, Indian Institute of Spices Research, India

Park, Jin-Woo

Department of Plant Pathology, National Institute of Agricultural Science and Technology, Korea

Cheon, Jeong-Uk

Divisioin of Crop Research, National Institute of Alpine Agriculture, Korea

他

https://doi.org/10.5109/4585

出版情報：九州大学大学院農学研究院紀要. 49 (2)，pp. 253-262，2004-10-01. Faculty of Agriculture, Kyushu University

バージョン :

権利関係 : 


\title{
Studies on Potato virus $Y$ Isolates Infecting Potato and Tobacco in Korea
}

\author{
Hong-Soo CHOI*, A-Ishwara BHAT ${ }^{1}$, Jin-Woo PARK ${ }^{2}$, Jeong-Uk CHEON ${ }^{3}$, \\ Jeong-Soo KIM ${ }^{4}$, Hanu-R PAPPU ${ }^{5}$, Jang-Kyung CHOI ${ }^{6}$ \\ and Yoichi TAKANAMI
}

\author{
Laboratory of Plant Pathology, Division of Plant Pathology and Pesticide Science, \\ Department of Applied Genetics and Pest Management, Faculty of Agriculture, \\ Kyushu University, 6-10-1, Hakozaki, Fukuoka 812-8581, Japan \\ (Received June 24, 2004 and accepted July 13, 2004)
}

\begin{abstract}
A survey of Potato virus $Y$ (PVY) was conducted in farmers' fields in 9 Korean provinces, Gyeonggi-do, Gangwon-do, Gyeongsangnam-do, Gyeongsangbuk-do, Jeollanam-do, Jeollabuk-do, Chungcheongnam-do, Chungcheongbuk-do and Jeju-do from 1998 to 2000 . One hundred and fifty nine samples from potato and tobacco were collected and analyzed. Based on ELISA results, three samples PVY-tob, PVY-pot-jj and PVY-pot-chw representing PVY ${ }^{\mathrm{N}}$ and PVYo, respectively, were further subjected to biological, serological and molecular analysis. Host range studies showed that PVY-pot-chw is symptomatically distinguishable from PVY-tob and PVY-pot-ji isolates, both of which were symptomatically indistinguishable. However, all the three isolates failed to infect 10 capsicum varieties systemically. Isolates PVY-tob and PVY-pot-jj reacted strongly with monoclonal antibody (MAb), anti-PVYN while PVY-pot-chw failed to react with the $\mathrm{MAb}$ but reacted strongly with anti-PVYo/c. Interestingly, both PVY-tob and PVY-pot-jj isolates did not react with anti-PVYo/c indicating that isolate PVY-pot-chw is serologically distinguishable from both PVY-tob and PVY-pot-jj. In order to ascertain the taxonomic status of the three isolates, the 3' untranslated terminal regions (3'-UTR) were sequenced. Alignment of the sequences revealed $99 \%$ sequence identity between PVY-tob and PVY-pot-jj, both of which had 78\% nt sequences identical to PVY-pot-chw. Phylogenetic analysis of the aligned 3'-UTRs clustered PVY-tob and PVY-pot-jj in the PVYN subgroup, and PVY-pot-chw in the PVY ${ }^{\circ}$ subgroup.
\end{abstract}

\section{INTRODUCTION}

Potato virus $Y$ (PVY) is a type member of the genus Potyvirus, family Potyviridae (Francki et al., 1991). The virus has flexuous particles of length $740 \mathrm{~nm}$ containing a single-stranded messenger sense RNA genome of about $10 \mathrm{~kb}$ and one open reading frame (Reichmann et al., 1992). The virus is transmitted by aphids in a non-persistent manner. PVY naturally infects potato, pepper, tobacco, tomato, and other solanaceous plant species causing important yield losses worldwide (de Bokx and Huttinga, 1981).

1 Division of Crop Protection, Indian Institute of Spices Research, India

.2. Department of Plant Pathology, National Institute of Agricultural Science and Technology, Korea

${ }^{3}$ Division of Crop Research, National Institute of Alpine Agriculture, Korea

4 Department of Horticultural Environment, National Horticultural Research Institute, Korea

5 Department of Plant Pathology 353 Johnson Hall WSU-Pullman, USA

- Department of Agricultural Biology, Kangwon National University, Korea.

* Corresponding Author (E-mail: hschoi@rda.go.kr) 
Based on symptoms and host species, PVY isolates have been classified into three distinct groups $\left(\mathrm{Y}^{\circ}, \mathrm{Y}^{\mathrm{N}}\right.$ and $\mathrm{Y}^{\mathrm{C}}$ ) (de Bokx and Huttinga, 1981). The PVYo isolates (common strain) cause mottling in tobacco and crinkling, rugosity or leaf drop streaks in potato. PVY isolates induce necrotic symptoms in tobacco and mild mottling in potato. The PVYc isolates cause a hypersensitive reaction in potato carrying the Nc resistance gene and induces mosaic or stipple streak in potato cultivars (de Bokx and Huttinga, 1981). In Korea, the distribution of PVY is correlated with the widespread occurrence of potato (Solanum tuberosum L.) and tobacco (Nicotiana tabacum L.). Potato and tobacco are naturally infected with PVY, with infection rates ranging from $3.7 \%$ to $12.7 \%$ (La, 1974; Lee, 1981; Park et al., 1984). In potato, two PVY strains (PVY ${ }^{\circ}$ and PVYN) were identified in Daegwallyeong, an alpine area, using indicator plants. Comparison of the CP sequences revealed 97.3\% identity between PVY-O (Korea) and PVY-O (Japan) (Cheong et al., 1992). In addition to the biological and serological data, nucleotide sequence identities of the coat protein (CP) or 3'-UTR have been increasingly used in recent years to differentiate distinct species from strains (Shukla and Ward, 1989; Frenkel et al., 1989). Further, van der Vlugt et al. (1993) reported the subgrouping of PVY isolates based on the CP and 3'-UTR sequence identities that matched groupings based on biological and/or serological criteria. Although the occurrence of PVY on many crops is reported from South Korea, they have not been adequately characterized. In this study we report the results of a survey of PVY strains and their characterization based on biological, serological and 3'-UTR sequence properties.

\section{MATERIALS AND METHODS}

\section{Sample collection}

During May and October of 1998-2000, a survey of PVY was conducted in main potato and tobacco growing provinces of Korea namely, Gyeonggi-do, Gangwon-do, Gyeongsangnam-do, Gyeongsangbuk-do, Jeollanam-do, Jeollabuk-do, Chungcheongnam-do, Chungcheongbuk-do and Jeju-do (Table 1). The survey was conducted by walking through potato and tobacco fields, while inspecting the crops for PVY disease symptoms. Farmers in the respective fields were interviewed on the potato/tobacco variety grown, source of planting material, cultural practices and presence of other diseases. Disease incidence was calculated as the number of plants showing PVY symptoms relative to the total number of plants observed in an area.

\section{Symptom reproduction under controlled environment}

To determinate the infectivity of virus isolates and the symptoms induced on the test plant, 5-10 plant seedlings of each of the species, Chenopodium amaranticolor, $C$. quinoa, Nicotiana benthamiana, N. tabacum cv. 'Bright yellow', $N$. tabacum cv. 'Xanthi-nc', N. tabacum cv. 'Samsun', Physalis floridana, Petunia spp. at the 3-5 leaf stage were inoculated by sap extracted in $0.1 \mathrm{M}$ phosphate buffer, $\mathrm{pH}$ 7.0. The plants were put in an insect-free greenhouse maintained at $20-25^{\circ} \mathrm{C}$ with $12 \mathrm{~h}$ light period. Disease symptoms were recorded three times a week for 30 days. 


\section{DAC-ELISA}

Because polyclonal antibodies cannot be used to differentiate PVY strains due to the high level of epitope conservation on the CPs (Shukla and Ward, 1989), a PVY specific monoclonal antibodies were used. Direct-antibody coated enzyme linked immunosorbent assay (DAC-ELISA) was conducted essentially as described by Clark and Bar-Joseph (1984) using monoclonal antibodies (MAb) purchased from Adgen (United kingdom). The MAbs and conjugate were both diluted 1:1,000 and all incubations were carried out at $37^{\circ} \mathrm{C}$ for $2 \mathrm{~h}$ except for the substrate which was incubated for $30 \mathrm{~min}$. Quantitative measures of generated $p$-nitrophenol were made by determining absorbance at $405 \mathrm{~nm}\left(\mathrm{~A}_{405}\right)$ in an EL312e EIA model spectrophotometer (Bio-Tek Instruments Inc., Winooski, VT, USA). Twice absorbance value was considered positive to negative control.

\section{Electron microscopy}

Two weeks after inoculation, infected leaves were harvested and immediately cut with a sharp blade (which was washed with $75 \%$ ethanol after every cut) into $1-3 \mathrm{~mm}$ thick pieces. The newly cut pieces were immediately fixed with $2.5 \%$ glutaraldehyde in Millonig's phosphate buffer, $\mathrm{pH} 7.0$ at $4^{\circ} \mathrm{C}$. The pieces were then thoroughly rinsed in Millonig's phosphate buffer before being fixed with $2 \%$ osmium tetraoxide for $90 \mathrm{~min}$. The pieces were then stained overnight in $1.0 \%$ uranylacetate at $4{ }^{\circ} \mathrm{C}$ and then rinsed in distilled water. The pieces were dehydrated six times each with 50-100\% ethanol for 50 min. The dehydrated pieces were embedded in Spur resin and hardened overnight at $60^{\circ} \mathrm{C}$. Ultra-thin sections of $80 \mathrm{~nm}$ thickness were sliced using ultramicrotome and a knife. The sections were then stained twice, with $2 \%$ uranylacetate for $20 \mathrm{~min}$ then with $0.5 \%$ lead citrate for $10 \mathrm{~min}$. For interpretation of results, the sections were observed under an electron microscope LEO $912 \mathrm{AB}$ at $80 \mathrm{kV}$.

\section{RT-PCR}

Total RNA was extracted from infected leaf samples essentially as described by Prescott and Martin (1987). The 3'-terminal region of both potato and tobacco isolates of PVY comprising part of the coat protein (CP) gene and the 3'-UTR was amplified following the RT-PCR procedure described by Pappu et al. (1993) using primers listed in Table 2.

\section{Cloning and sequencing}

The amplified PCR products were cloned in pGEM-T vector (Promega) and sequenced by a commercial company (Green gene biotechnology, Korea). Potato/tobacco PVY sequences obtained were phylogenetically compared to those of other PVY (GenBank and EMBL) using the multiple sequence alignment application of DNAMAN version 4.0 (Lynnon Biosoft, Quebec, Canada) full optimal sequence alignments and neighbor-joining method options of Saitou and Nei (1987) with 100 bootstrap (Felstein, 1985) replications. Percent nucleotide (nt) and ORF amino acid (aa) sequence identities between virus isolates were calculated using the distance between all pairs of sequences in the multiple alignments. PVY (CP-UTR) sequences used for comparison and their database accession numbers were as follows: AJ223592 (PVY-N854 isolate), AJ390285 (PVY-N-RB isolate), AJ390295 (PVYN-S-NTN isolate), D12570 (necrotic-PVY-T 
isolate), X97895 (N605 isolate), Z70237 (Nysa isolate), U09508 (N27-92 isolate), AJ223593 (O768 isolate), D12539 (PVY-O isolate), U09509 (PVYO-Canadian isolate), AJ390289 (v942490 isolate), U10378 (Hungary pepper isolate), M22470 (N-PVY isolate), M95491 (Hungarian isolate).

\section{RESULTS}

\section{Sample collection and analysis}

A total of 139 potato and 20 tobacco leaf samples from 240 farmers' fields were collected and analyzed. Both indicator host species and DAC-ELISA revealed that $84.3 \%$ of the 159 samples were infected with $\mathrm{PVY}^{\mathrm{N}}$ strain, $13.8 \% \mathrm{PVY}^{\mathrm{O}}$ strain and $1.9 \%$ had a mixed infection of both strains. Crosswise, $83.5 \%$ of potato samples were infected with $\mathrm{PVY}^{\mathrm{N}}, 15.8 \%$ with $\mathrm{PVY}{ }^{\circ}$ and $0.7 \%$ with both $\mathrm{PVY}^{\mathrm{N}}$ and $\mathrm{PVY}^{\circ}$ strains. Tobacco had a higher $\mathrm{PVY}^{\mathrm{N}}$ infection of $90.0 \%$ and only $10 \%$ of the samples were infected with $\mathrm{PVY}^{\mathrm{o}}$. A striking feature in tobacco was that there was no double infection of both strains (Table 1). Of all the plants tested only those in the family Solanaceae were susceptible to PVY (Table 2). In N. tabacum, PVY-tob and PVY-pot-jj isolates caused vein clearing and vein necrosis but PVY-pot-chw induced mild mosaic symptoms in addition to vein clearing. It is worth noting that all the three strains failed to infect and induce symptoms in Sesamum indicum L., Phaseolus vulgaris and Datura stramonium (Table 2). Of the 10 varieties of Lycopersicon esculentum tested, 6 were found to be susceptible to both PVY-pot-chw and PVY-pot-ij isolates while only 1 was susceptible to PVY-tob. However, all the 10 cultivars of Capsicum annum were found to be immune to the strains as no virus was detected in the plants by either ELISA and/or electron microscopy (Table 3).

Table 1. Results of a survey of PVY in 9 provinces in Korea.

\begin{tabular}{llcccc}
\hline \multirow{2}{*}{ Host } & \multirow{2}{*}{ Provinces } & No. of & \multicolumn{3}{c}{ PVY strain ${ }^{\text {b) }}$} \\
\cline { 4 - 6 } detected plant & & N+O/C & N & O/C \\
\cline { 3 - 6 } Potato & Gangwon-do & 3 & 0 & 2 & 1 \\
& Gyeonggi-do & 10 & 0 & 8 & 2 \\
& Gyeongsangnam-do & 25 & 0 & 19 & 6 \\
& Gyeongsangbuk-do & 11 & 0 & 10 & 1 \\
& Jeollanam-do & 26 & 1 & 19 & 6 \\
& Jeollabuk-do & 7 & 0 & 7 & 0 \\
& Chungcheongnam-do & 22 & 0 & 20 & 2 \\
& Chungcheongbuk-do & 23 & 0 & 19 & 4 \\
& Jeju-do & 12 & 0 & 12 & 0 \\
\hline \multirow{2}{*}{ Tobacco } & Chungcheongbuk-do & 7 & 2 & 5 & 0 \\
& Chungcheongnam-do & 2 & 0 & 2 & 0 \\
& Jeollabuk-do & 11 & 0 & 11 & 0 \\
\hline
\end{tabular}

a) estimated by visual inspection

b) estimated by ELISA 
Table 2. Symptoms developed on indicator plants inoculated with PVY-pot-chw, PVY-pot-jj and PVY-tob isolates.

\begin{tabular}{|c|c|c|c|}
\hline \multirow{2}{*}{ Indicator plant test } & \multicolumn{3}{|c|}{ PVY Isolate } \\
\hline & PVY-pot-chw & PVY-pot-jj & PVY-tob \\
\hline Chenopodium amaranticolor & $-1-(\mathrm{cl} /-) \mathrm{a}$ & $-/-(\mathrm{cl}, \mathrm{nl} /-)$ & $-/-(\mathrm{cl} /-)$ \\
\hline C. quinoa & $\mathrm{nl} /-$ & $\mathrm{nl} /-$ & $\mathrm{nl} /-$ \\
\hline Nicotiana benthamiana & $\mathrm{cl} / \mathrm{cl}, \mathrm{sM}$ & $\mathrm{cl} / \mathrm{cl}, \mathrm{sM}$ & $\mathrm{cl} / \mathrm{cl}, \mathrm{sM}$ \\
\hline N. tabacum cv. 'Bright yellow' & $\mathrm{cl} / \mathrm{vc}, \mathrm{mM}$ & $\mathrm{nl} / \mathrm{nl}, \mathrm{vn}$ & $\mathrm{nl}, \mathrm{vn} / \mathrm{nl}, \mathrm{vn}$ \\
\hline N. tabacum cv. 'Xanthi-nc' & $\mathrm{cl} / \mathrm{vc}, \mathrm{mM}$ & $\mathrm{nl} / \mathrm{nl}, \mathrm{vn}$ & $\mathrm{nl}, \mathrm{vn} / \mathrm{nl}, \mathrm{vn}$ \\
\hline N. tabacum cv. 'Samsun' & $\mathrm{cl} / \mathrm{vc}, \mathrm{mM}$ & $\mathrm{nl} / \mathrm{nl}, \mathrm{vn}$ & $\mathrm{nl}, \mathrm{vn} / \mathrm{nl}, \mathrm{vn}$ \\
\hline Physalis floridana & $\mathrm{cl} / \mathrm{cl}, \mathrm{M}$ & $\mathrm{nl} / \mathrm{nl}, \mathrm{vn}$ & $\mathrm{cl} / \mathrm{cl}, \mathrm{M}$ \\
\hline Petunia spp. & $\mathrm{cl} / \mathrm{m}$ & $\mathrm{cl} / \mathrm{vc}, \mathrm{vn}$ & $\mathrm{nl} / \mathrm{vn}$ \\
\hline Datura stramonium & $-1-$ & $-1-$ & $-1-$ \\
\hline Tetragonia expansa & $\mathrm{cl} /-$ & $\mathrm{cl} /-$ & $\mathrm{cl} /-$ \\
\hline Datura stramonium & $-1-$ & $-1-$ & $-1-$ \\
\hline Sesamum indicum L. & $-1-$ & $-1-$ & $-1-$ \\
\hline Perilla frutescens & $-1-$ & $-1-$ & $-1-$ \\
\hline Impatiens balsamina $\mathrm{L}$ & $-1-$ & $-1-$ & $-1-$ \\
\hline Zinnia elegans Jacq. & $-1-$ & $-1-$ & $-1-$ \\
\hline Cucumis sativus L. & $-1-$ & $-1-$ & $-1-$ \\
\hline C. melo L. & $-1-$ & $-1-$ & $-1-$ \\
\hline Citrullus lanatus & $-1-$ & $-1-$ & $-1-$ \\
\hline Cucurbita moschata Duch & $-1-$ & $-1-$ & $-1-$ \\
\hline Raphanus sativus L. & $-1-$ & $-1-$ & $-1-$ \\
\hline Brassica campestris & $-1-$ & $-1-$ & $-1-$ \\
\hline B. $\operatorname{rapa} \mathrm{L}$ & $-1-$ & $-1-$ & $-1-$ \\
\hline Chrysanthemum coronarium & $-1-$ & $-1-$ & $-1-$ \\
\hline Glycine $\max$ Merr. & $-1-$ & $-1-$ & $-1-$ \\
\hline Phaseolus vulgaris L. & $-1-$ & $-1-$ & $-1-$ \\
\hline P. angularis & $-1-$ & $-1-$ & $-1-$ \\
\hline$P$. radiatus $\mathrm{L}$. & $-1-$ & $-1-$ & $-1-$ \\
\hline Vicia faba & $-1-$ & $-1-$ & $-1-$ \\
\hline Vigna sinensis King & $-1-$ & $-1-$ & $-1-$ \\
\hline
\end{tabular}

${ }^{a} \mathrm{cl}$, chlorotic local; nl, necrotic local; $\mathrm{mM}$, mild mosaic; $\mathrm{m}$, mosaic; sM, severe mosaic; vc, vein clearing; vn, vein necrosis; inoculated leaves/upper leaves; -, no symptoms.

\section{Serological relationships}

Both PVY-tob and PVY-pot-jj isolates reacted strongly with anti- PVYN serum but failed to react with anti-PVYoc while PVY-pot-chw isolate reacted strongly with anti-PVY ${ }^{a c}$ but failed to react with anti- PVYN. Therefore PVY-tob and PVY-pot-jj are serologically distinguishable from PVY-pot-chw.

\section{Electron microscopy}

Examination of the thin sections from the infected potato and tobacco revealed typical cytoplasmic inclusions bodies like pinwheels, scrolls, laminated aggregates and tubes in cells of $N$. tabacum cv. 'Xanthi-nc' (Fig. 1). Additionally, many virus particles were spotted around the mitochondria. Crude sap observation revealed long flexuous particles of $750 \mathrm{~nm}$ in length, but no inclusion bodies. 
Table 3. Infectivity of PVY-pot-chw, PVY-pot-jj and PVY-tob isolates in tomato and red pepper cultivars.

\begin{tabular}{ccccc}
\hline \multirow{2}{*}{ Indicator plant test } & \multicolumn{3}{c}{ PVY Isolate } & \\
\cline { 2 - 4 } Lycopersicon esculentum cv & PVY-pot-Chw & PVY-pot-Jj & PVY-tob \\
'Dotaerang B' & 'Dotaerang' & + a & + & + \\
'House dotaerang' & + & + & + & - \\
'Yeonggwang' & + & + & - \\
'Seogwang' & + & + & - \\
'Ponterosa' & + & - & - \\
'Youngmuja' & + & + & - \\
'Minicarol' & - & - & - \\
'PePe' & - & - & - \\
'Yoyo' & - & - & - \\
\hline Capsicum annuum cv & - & - & - \\
'Dongbang' & - & - & - \\
'Geosung' & - & - & - \\
'Pungchon' & - & - & - \\
'Gumtop' & - & - & - \\
'Daewon' & - & - & - \\
'Daemung' & - & - & - \\
'Joyang' & - & - & - \\
'Chungok' & - - & - & - \\
\hline
\end{tabular}

a + , symptoms; -, no symptoms.

Table 4. Primers used for amplification of the 3'-terminal untranslated region (3'-UTR) of PVY isolates from Korea.

\begin{tabular}{cll}
\hline Primer & \multicolumn{1}{c}{ Sequence $^{\text {a }}$} & \multicolumn{1}{c}{ Primer Position } \\
\hline HRP 1 & 5'TTT TTT TTT TTT TTT TTT TTT A3' & poly A tail \\
HRP 2 & 5'TTT TTT TTT TTT TTT TTT TTT G3' & poly A tail \\
HRP 3 & 5'TTT TTT TTT TTT TTT TTT TTT C3' & poly A tail \\
HRP 4 & 5'TGG TGY ATH GAN AAT GG3' & CP (conserved WCIEN region) \\
\hline
\end{tabular}

a Primers designed by Pappu et al. (1993); N=A, C, G, T; Y=C, T; H=A, T, C.

\section{3'-UTR sequence comparison with known PVY isolates}

The PVY-pot-chw's 3'-UTR sequenced was found to contain 720 nucleotides (nts) including part of the CP of 150 amino acids, while isolates PVY-tob and PVY-pot-jj each contained 325 nts. A detailed analysis of the aligned 3'-UTR nucleotide sequences revealed a varying degree of sequence identity with isolates PVY-tob and PVY-pot-jj sharing $99 \%$ nt and only $89 \%$ identity with isolate PVY-pot-chw. A pair-wise comparison 


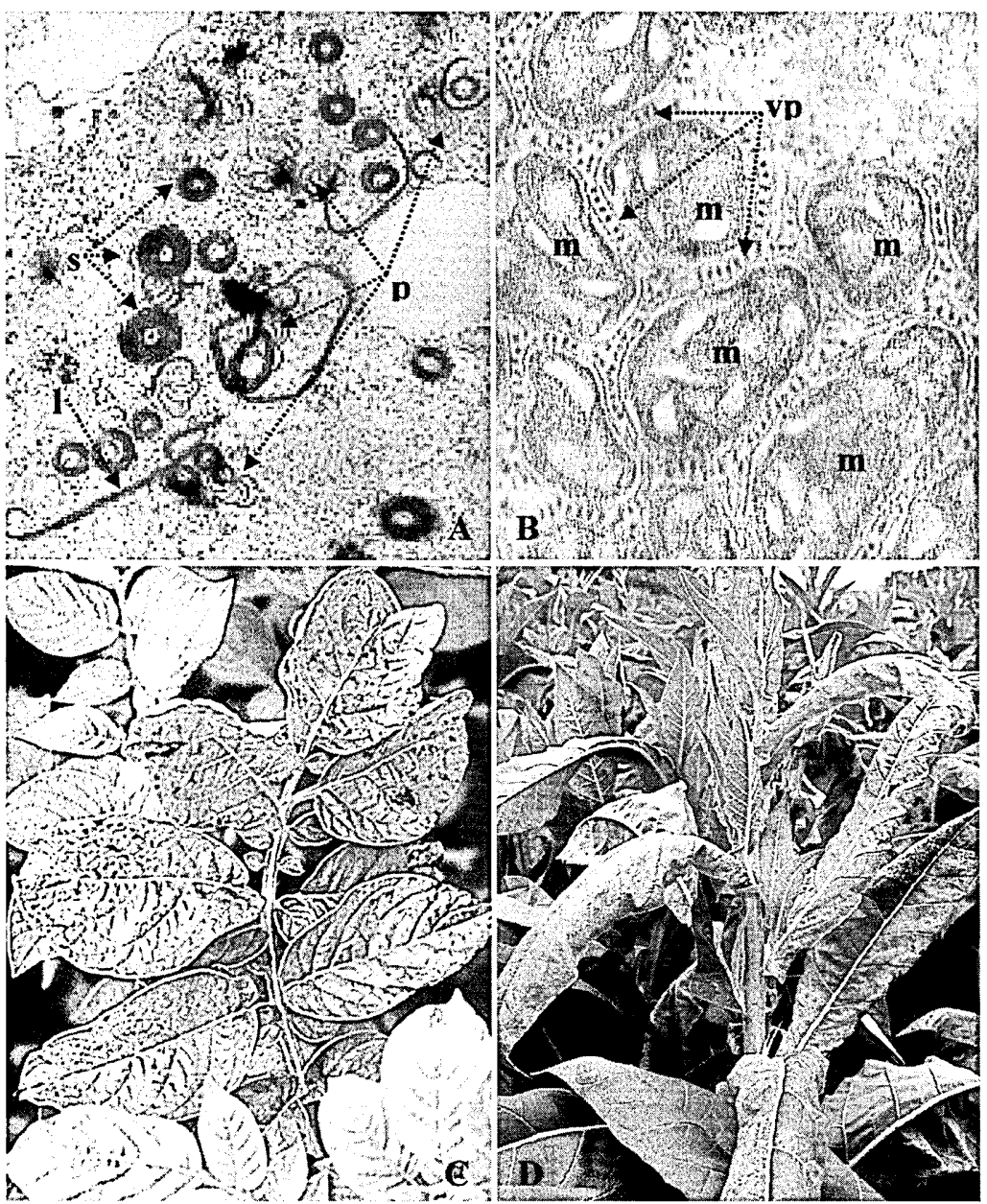

Fig. 1. Typical cytoplasmic inclusions bodies like pinwheels, scrolls, laminated aggregates and tubes (A) and virus particles around the mitochondria (B) in cells of Nicotiana tabacum cv. 'Xanthi-nc'. Potato plants (C) showing necrotic symptoms by PVY-pot-jj isolate and tobacco plants (D) showing vein clearing and necrotic symptoms caused by PVY-tob isolate in the fields. m, mitochondria; vp, virus particles; s, scrolls; l, laminate aggregates; $p$, pinwheels.

of the 3'-UTR sequences of PVY-tob, PVY-pot-jj, and PVY-pot-chw isolates with those of the other PVY isolates from the database revealed 76-99\% sequence identity (data not shown). Phylogenetic analysis of the aligned sequences revealed a tree structure whose clusters defined separate groups with both PVY-pot-jj and PVY-tob isolates falling within the $\mathrm{PVY}^{\mathrm{N}}$ group while potato-chw fell within the $\mathrm{PVY}{ }^{\mathrm{O}}$ group (Fig. 2). 


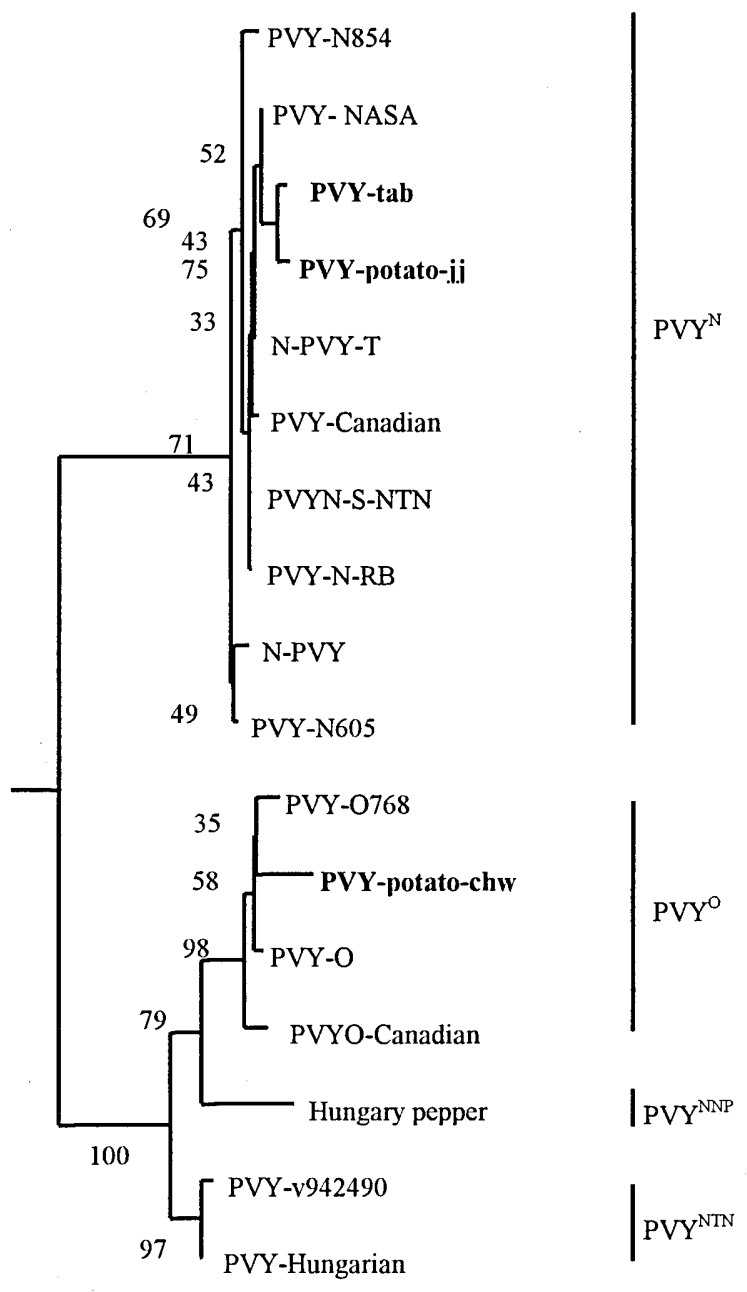

Fig. 2. Phylogenetic tree constructed from nucleotide sequence alignments of the 3'-UTR fragments of PVY isolates from tobacco and potato.

\section{DISCUSSION}

Symptoms of PVY-pot-jj and PVY-tob such as necrotic spots, vein and petiole necrosis indicated that the isolates belonged to the necrotic strains group, while PVY-pot-chw isolate that exhibited vein clearing and mild mosaic belonged to the common strain group. An interesting observation is that all isolates failed to infect $C$. annuum, although it contradicts the finding of Lee (1981) that PVY infects C. annuum. 
Nevertheless, 6 of the 10 varieties of $L$. esculentum tested were infected with both PVY-pot-chw and PVY-pot-jj while PVY-tob infected only one variety of L. esculentum. To reveal the exact identity of these isolates, their 3'-UTRs were sequenced and compared with those of 15 distinct PVY isolates. High degree of 3'-UTR sequence identities (84-99\%) of both PVY-pot-jj and PVY-tob isolates with known PVY isolates confirmed them as strains of PVY as they were within the cut-off range of $83-99 \%$ for identifying strains of the same virus (Frenkel et al., 1989). The 3'-UTR sequences of PVY-pot-jj and PVY-tob isolates differed from those of known tobacco vein necrotic strains of PVY occurring in Korea (PVY-VN). This possibly indicates the existence of variation in the necrotic isolates of PVY within the country. The 3'-UTR has been shown to regulate symptom severity (Rodriguez-Cerezo et al., 1991). Further studies involving additional PVY isolates representing different agro-climatic regions of the country are required in order to detect and identify the existence of various distinct PVY isolates. This would help in developing an effective strategy for managing the viruses.

\section{ACKNOWLEDGEMENTS}

We thank Dr. H. K. Were for his valuable suggestions and the critical reading of the manuscript. We are also grateful to Dr. M. Takeshita for very useful advice, and Mr. J. S. Lee for assistance with the electron microscopic work.

\section{REFERENCES}

Cheong, S. R., T. Goto, Y. I. Hahm and J. K. Choi 1992 A strain of potato virus Y (PVY) isolated from potato plant in Korea. Korean J. Plant Pathol., 8: 209-212

Clark, M. F. and M. Bar-Joseph 1984 Enzyme immunosorbent assays in plant virology. Methods Virol., 7: $51-85$

de Bokx, J. A. and H. Huttinga 1981 Potato virus Y. CMI/AAB Descriptions of Plant Viruses No. 242

Felsenstein, J. 1985 Confidence limits on phylogenies: An approach using the bootstrap. Evolution 39: 783-791

Francki, R. I. B., C. M. Fauquet, D. L. Knudson and F. Brown 1991 Classification and Nomenclature of Viruses: Fifth Report of the International Committee on Taxonomy of Viruses. Arch. Virol. (Suppl) 2

Frankel, M. J., C. W. Ward and D. D. Shukla 1989 The use of 3,-noncoding nucleotide sequences in the taxonomy of potyviruses: application to watermelon mosaic virus-2 and soybean mosaic. J. Gen. Virol., 70: 2775-2783

La, Y. J. 1974 Serological identification of potato viruses in Korea. Korean J. Plant Protect., 14: 41-45

Lee, S. H. 1981 Studies on virus disease occurring in various crops in Korea. Res. Rept. RDA, 23: 62-74

Pappu, S. S., A. Brand, H. R. Pappu, E. P. Rybicki, K. H. Gough, M. J. Frankel and C. L. Niblett 1993 A polymerase chain reaction method adopted for selective amplification and cloning of 3'-sequences of potyviral genomes: application to dasheen mosaic virus. J. Virol. Methods, 41: 9-20

Park, E. K., J. J. Kim and K. S. Boo 1984 Two new PVY strains isolated from tobacco plant in Korea. Korean J. Plant Protect., 23: 209-214

Prescott, A. and C. Martin 1987 A rapid method for the quantitative assessment of levels of specific mRNAs in plants. Plant Mol. Biol. Rep., 4: 219-224

Reichmann, J. L., S. Lain and J. A. Garcia 1992 Highlights and prospects of potyvirus molecular biology. J. Gen. Virol., 73: 1-16

Rodriguez-Cerezo, E., P. G. Klein and J. G. Shaw 1991 A determinant of disease symptom severity is located in the 3'-terminal noncoding region of the RNA of a plant virus. Proc. Nat. Acad. Sci. USA, 88: $9863-9867$

Saitou, N. and M. Nei 1987 The neighbor-joining method: a new method for reconstruction of 
phylogenetic trees. Mol. Biol. Evolut., 4: 406-425

Shukla D. D. and C. W. Ward 1989 Identification and classification of potyviruses on the basis of coat protein sequence data and serology. Arch. Virol., 106: 171-200

Van der Vlugt, R. A. A., J. Leunissen and R. Goldbach 1993 Taxonomic relationships between distinct potato virus $Y$ isolates based on detailed comparisons of the viral coat proteins and 3'-nontranslated regions. Arch. Virol., 131: 361-375 